\title{
A new plasmonics enhanced ultrafast laser multi-nanoscapel (Presentation Video)
}

\section{Michel Meunier}

Michel Meunier, "A new plasmonics enhanced ultrafast laser multi-nanoscapel (Presentation Video)," Proc. SPIE 8972, Frontiers in Ultrafast Optics:

Biomedical, Scientific, and Industrial Applications XIV, 89721H (18 March 2014); doi: $10.1117 / 12.2063584$

SPIE. Event: SPIE LASE, 2014, San Francisco, California, United States 


\title{
A New Plasmonics Enhanced Ultrafast Laser Multi-Nanoscapel (Presentation Video)
}

\author{
Michel Meunier, Ecole Polytechnique de Montreal (Canada)
}

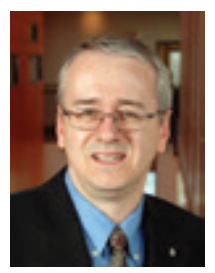

\begin{abstract}
A new technique is introduced to perform nanosurgery in living cells using a laser multi-nanoscapel. Irradiating plasmonics nanostructures by an ultrafast laser beam produces highly localised processes on the nanoscale in the biological surrounding medium, yielding to the nanosurgery of cells. These nanoparticles could be functionalised to target specific biological entities, thus performing multiple targeted surgeries on the nanoscale. As an example, the laser multi-nanoscapel was employed to perform gene transfection in living cell with an optoporation efficiency as high as $70 \%$. Complete physical model was developed to determine the basic mechanism underlying this new nanosurgery process. Our laser multi-nanoscapel shows promises as an innovative tool for fundamental research in biology and medicine as well as an efficient alternative nanosurgery technology that could be adapted to therapeutic tools in the clinic.
\end{abstract}

View presentation video on SPIE's Digital Library: http://dx.doi.org/10.1117/12.2063584 